\title{
The Innovating Method of Measuring the Symbolic Capital of Youth
}

\author{
Filip Nalaskowski, Dagna Dejna \\ Uniwersytet Mikołaja Kopernika, Torun, Poland
}

\begin{abstract}
The paper presents an original and innovative method of measurement of symbolic capital, which is the final instance of conversion of an economic, cultural, and social capital. The method of measurement has been created by the authors of this study. The methodological path of the research is theoretically founded in the concept of Pierre Bourdieu, a French sociologist and anthropologist. A tool for measurement of the level of capitals among young people as used in the text makes it possible to explain complex social and demographic processes and deep changes in the picture of contemporary societies. This method may also be used for formulation of requests relating to political choices and preferences of young people, changes in customs, dynamics of social structures and stratification processes. In detailed description of the research tool, research method, and theoretical issues, the authors present their two own research projects, in realisation of which they used Pierre Bourdieu's concept and their own concept of measurement of capitals.
\end{abstract}

Keywords: Bourdieu's concept, symbolic capital, the authors' own method of measurement, young people, research

In the paper, it will present an original and innovative way of measuring of the capitals: economic, social, and cultural, which make up the symbolic capital. The authors are members of the research team of young university dormitories of Torun city- $\mathrm{ACCEPT}^{1}$. It was the team who came up with the idea of the study of symbolic capital, which has its origins in the concept of the eminent French sociologist and philosopher-Pierre Bourdieu.

The presented strategy research procedure was subjected to numerous tests, and proved its usefulness in practice. What distinguishes the approach is that you can, by relatively little effort and resources, using a simple tool, obtain information about the approximate level of individual capitals.

Above all, this tool developed by the authors to measure the levels of capitals using the theory of $\mathrm{P}$. Bourdieu is a great way of obtaining data from almost all areas of social functioning of individuals. Both the theory and one of the ways of its implementation, which the authors created, can be used to create new solutions in the field of social policy, education policy, public administration, and new social solutions.

The paper is divided into three main parts. In the first part, the authors reconstruct the intellectual sources of research strategies and theoretical contexts of understanding of the concept of capitals. The second part

Corresponding author: Filip Nalaskowski, Ph.D., educator, sociologist, Uniwersytet Mikołaja Kopernika; specialist in sociology of education and methodology of social research, founder and initiator of a research team-ACCEPT; research fields: sociology of education and methodology of social research. E-mail: filipn@umk.pl.

Dagna Dejna, Ph.D., educator, Uniwersytet Mikołaja Kopernika; specialist in general pedagogy and ethnopedagogy, research team member-ACCEPT; research fields: general pedagogy and ethnopedagogy. E-mail: dagnadejna@umk.pl.

${ }^{1} \mathrm{http} / / / \mathrm{www}$.accept.umk.pl. 
presents in detail the author's method, technique, and tool designed to measure the capitals, also describe the workflow of research on each of the necessary stages. The third part contains conclusions and analysis capabilities, and above all, a brief description of the two conducted by the authors large research projects in which the described procedure was used.

\section{Concepts of Capitals-Sources and Bourdieu Game}

As already mentioned above, in the presented author's strategy to measure capitals, the authors refer to the concept of P. Bourdieu (1930-2002) - internationally recognized as an eminent French sociologist, anthropologist, and philosopher. Since 1981, Bourdieu has been a member of the College de France, he was also the author of important for contemporary social science books: Invitation to Reflective Sociology, Refinement, Rules of Art, Men Domination. P. Bourdieu primarily dealt with research in the sociology of culture, education, and social science theory. The key to the issues in question is a book-Reproduction. Elements of the Theory of the Educational System is the study of the basic processes of social stratification and cultural transmission. Bourdieu analyzes the problems of education, showing what role it plays in the reproduction of the social structure, and above all in the transmission of culture to the next generation. It is in this book, Bourdieu describes in detail his own concept of symbolic capital and of subsequent capitals: economic, social, and cultural. This work, and thus the concept of a French sociologist, was the starting point for the presented in the text research approach.

Reproduction was issued by Bourdieu with a close collaborator on his several important books-Jean Claude Pearson. In this book, the authors analyze constructing and reproducing mechanisms of the social hierarchy of egalitarian, by definition, democratic society. Almost from the beginning of his scientific career, Bourdieu believed that the area in which societies form social distinctions is manufacturing field of culture. A person in Bourdieu participates in the social space consisting of different fields, multidimensional, and his/her position is determined not by class membership, but by the amount of capital held. Symbolic capital determines the difference, is also a mechanism for social mobility (Bourdieu, 2007).

The discussion around the concept of capitals is now taking place not only in the scientific literature, but also in journalism, media, and popular science literature. Unfortunately, the concept of social capital has become rapidly a buzzword a kind of fashionable ornament for rhetorical skirmishes.

Often, and wrongly, it is believed that the concept of social capital was popularized by texts of $\mathrm{P}$. Bourdieu and J. Coleman.

In fact, the first time this term was used by a pedagogue - Lynda J. Hanifan. Social capital is treated as a result of a multicultural historical process, conditioned by many parallel factors (Trutkowski \& Mandes, 2005).

After many years, since the introduction to the social sciences, the term "social capital", different schools still collide with each other. Some people consider social capital to be a dimension, which can be used to describe only individuals and small groups, others - that it applies to the whole of society. Parallel still is ongoing discussion on the relationship among economic, cultural, and social capital (Growiec, 2011).

Pierre Bourdieu called the capital an element, which in the most fundamental way organizes social life. He distinguished three forms of capital: economic, cultural, and social. To these three main forms, added symbolic and political capital: Symbolic: It is the same as the socially sanctioned and institutionally established power, which results from the collected resources associated with the three basic forms of capitals. Usually manifests 
itself as a prestige assigned to a particular social position, such as, for example, reputation. Political: a specific form of social capital that had to exist mainly in Soviet-type societies.

Economic capital is in the form of material resources. Cultural capital is understood as any kind of knowledge, learnedness, erudition, skills, level of cultural competence. Social capital in terms of Bourdieu represents,

The sum of resources, actual and potential, that belong to an individual or a group, from the ownership of non-volatile, more or less institutionalized network of relationships, knowledge and mutual recognition. It means that it is the sum of capital and power that such a network can mobilize.

According to this definition, the individual potential of social capital ownership, depends greatly on the range of networks and relationships that a person builds, and by which gains access to specific resources. The key role plays here, however, the size and quality of these resources, this is the total value of the capitals held by members of a social network (or a social group). According to this, it can be concluded that it is a specific combination, or the structure of certain forms of capital, which is the share of a person determines his/her position in a society.

Social capital possessed by a person depends so greatly on the effort that he/she puts in other forms of capital accumulation. Thus, the greater the economic resources and richer resource of cultural capital, the greater the chance of developing networks and related feasibility of collecting the desired resources, it should be noted, however, that a large social capital significantly facilitates accumulation of other forms of capital. In summary, social capital is a "multiplier" of other forms of capital, while it is produced and sustained by the conversion of cultural and economic capital (Trutkowski \& Mandes, 2005).

In contrast, the aforementioned Coleman's concept is considered to be a classic for a holistic and systematic approach to social capital, and therefore should, at this point be briefly mentioned. It had originally helped in explaining school success and failures of children raised in different families and social environments. Coleman says, which is particularly relevant to debated issues that social capital is a set of resources that are included in family relationships, or that result from specific relationships and social relations in any community. These resources support the cognitive and social development of man; they vary depending on the environment of origin. Coleman treats the social capital as the main factor that determines how human capital will be ultimately invested and used. Coleman outlines, that social capital consists of two components. Firstly, it is characteristic of the social structure (the system of relations). Second, it facilitates some personal actions, undertaken within this structure. Social capital means the resources, actual or potential, generated only by the existence between persons, different types of elations and connections.

Coleman also argues that the key here is "closing" the network of social relations, because the closure of the network is a prerequisite for developing and maintaining the standards that apply within it. It also determines whether the trust in the given relationship network reaches a sufficiently high level.

Another factor contributing to the reproduction of social capital is the stability of the social structure. According to Coleman, each form of social capital is dependent on the stability of the network of relationships, and any disorder in this stability is destructive.

The third factor in the formation of social capital is the ideology, and, more generally speaking - normative code. It determines whether or not an individual, having available significant capital resources will also be willing to undergo a normative requirement to act altruistically. 


\section{Methodological Issues}

Lack of agreement among researchers regarding the definition of social capital, unfortunately causes difficulties in the study of this capital. The authors struggled with these difficulties, when they were planning the presented research project. Firstly, the very choice of indicators and scales created for them may raise methodological doubts. For example, Robert D. Putman as indicators of social capital assumed both variables related to social networks (social networks), and participation in local government organizations, as well as the norms of trust and reciprocity. Difficulties in the study of capital are also linked to the fact that in the debates about them there is a normative aspect.

In designing the study, we used the concept of P. Bourdieu, based on the main thesis contained in the book Reproduction. Elements of the Theory of the Educational System. Bourdieu argues that the social world cannot be reduced only to the circulation of economic capital. The starting point for Bourdieu was inequality of educational achievements of children, which cannot be explained only by the economic capital of parents. This researcher has developed the concept of cultural reproduction, which enforces social reproduction-governs relations between groups and social classes.

According to P. Bourdieu's theory, symbolic capital is the final court of conversion of cultural, social, and economic capitals (Bourdieu, 1986). Each of these different forms of capitals, we understood as a real resource and form of power. Economic capital can be immediately and directly converted into money. It is also institutionalized in the form of ownership. Cultural capital can, under certain conditions, be exchanged for economic capital and is institutionalized in the form of educational competence. It also determines the standards of behavior and good taste. In contrast, social capital is created from social obligations, so-called relationship. Social capital in Bourdieu's concept serves as a "multiplier" of other forms of capital, and allows to achieve higher profits from them. Between different forms of capital may occur conversion (Growiec, 2011). It is worth noting that, according to Bourdieu, with similar resources of cultural and economic capital, the social capital is a factor that makes a difference. Social capital is the capital of belonging, recognition, and authentication received from members of the group to which we belong, or to which we usurp a membership. Bourdieu was interested in how different types of capital together distinguished "living conditions main classes" on the basis of the different distribution of their total capital among the various types of capitals (Bourdieu, 1986).

\section{Research-Basic Information}

The strategy proposed below underwent a number of tests, and proved its usefulness in practice. What distinguishes this approach is that with relatively little effort and resources, using a simple tool, the authors can obtain information about the approximate level of the capitals. The specificity of the approach presented here directs the study of young people - for example, high school seniors, students, and young workers. Roughly, we can assume people aged 15-25 years.

Research of symbolic capital is usually only part of the whole diagnosis. This in itself cannot be autotelic. Useful information, new knowledge, we will build by the correlations of symbolic capital with other facts. One can ask, what is the relationship of capitals with gender, place of residence, family income, or even the average daily temperature. Recognition of capitals should be a part of the research problem.

\section{Method, Technique, Tool}

In the proposed approach, the authors use the method, the questionnaire technique. This strategy is 
supported by several arguments. Firstly, such studies provide fast effect of the requested information, and their analysis and processing is relatively quick and easy. In general, the work after collecting data is to encode and process this data.

As signaled earlier this form of searching of information does not require the involvement of the extraordinary manpower and resources. To collect the data, a small team is sufficient, and the cost is limited to printing questionnaires and to respondent search.

The last argument matter is the fact that the surveys are probably the most easily acceptable by the school authorities and large institutions gathering young people. It is from well-known and not arising controversy (as opposed to, for example, experiments). It is not a huge complication for a school "business day"-because it takes a few minutes deducted from a lesson hour or from a break.

What is also very important for the directors of these institutions - such a test is quite easy for them to control. The questionnaire leaves no doubt as to the intentions and the test area, and the presence of local observers (for example, teachers, educators), it is natural in the process of research, and gives an additional guarantee of proper conduct.

Therefore, the proposed design of the research tool questionnaire would be as follows.

\section{The Section on Measurement of Symbolic Capital}

For maximum simplicity and ease of participation, but also for analyzes the nature of the questions should be closed, and the entire questionnaire should take the focused form and fit on 1-2 A4 sheets.

Questions from the first part will focus on the register characteristics of the respondents. In this section, the authors traditionally ask, for example, about age, gender, place of residence of the respondents, or the school they attended.

Another part of the questionnaire should include the main research problem on which the authors focus. Here thay are talking about the subject of study which will be supplemented by information on the amount of capitals. In educational research that the authors conduct, these problems relate, for example to: education plans, career aspirations, relation to the state and society. It should be emphasized that from the point of view of interest to us here symbolic capital measurement, in the middle section, may be found, in principle, any social, educational problem. Importantly, in certain cases, we can swap places of Part 2 with Part 3, in order to first recognize the capital, and then the main problem.

The third, large components of the questionnaire are questions to measure the symbolic capital, according to the described earlier, concept of P. Bourdieu. The purpose of this component is to assess the levels of capitals: economic, social, and cultural in a simple and clear way. Obviously, in so simplified diagnosis, possible is only an approximation of the value of each capital. Therefore, a selection of questions was careful and should be tested in the pilot studies. The proposed questions may vary depending on the environments and cultures in which the study is carried out. It is prior to the study to carefully analyze the various threads and, if necessary, regionalize tool in order to better adapt it to the test environment.

The partial capital level is estimated on a scale of 0 to 10 points in the case of economic and social capital and cultural capital of 0 to 11 points - the larger the sum, the "richer" is a person symbolically. Different themes and questions are designed in close relation to the concept and guidelines of P. Bourdieu. In addition, the authors were looking for support in this area at the experiences of other researchers, constructing similar tools, conducting similar studies. 
Economic capital is diagnosed based on questions about the material level of families, from which come the respondents, but also about taken by themselves extra works. For these two questions, respondents could earn up to 10 points. The authors assume that on the economic capital of young people will have a strong impact from the material condition of the family of origin, and therefore statements concerning families are scored on a scale from 0-8, and taken by the respondents works only on a scale 0-2 (depending on the amount of income). Such a high value placed here on families stems from the fact that the study is addressed to young people who have not yet had a chance to build their own substantial financial resources and inevitably are dependent on the resources of their families.

Using the three questions, the authors try to assess the level of social capital. They assumed that in the very simple estimation, they will ask for access to specific services and professions, placed high on the social ladder. All questions have a similar structure. The respondents are placed in hypothetical situations- - health problems, legal problems, financial problems - and asked about whose help would they seek in such situations. If the answers show that in the circle of their friends are lawyers, doctors, people are willing to lend, then respondents obtain the maximum number of points (4-3-3 respectively). If the respondents are planning just to go to "non-experts" friends, the families, then they gain fewer points-one. If they do not have among friends people to whom they could go for help—do not get points. For this category, you can also obtain a maximum of 10 points.

The level of cultural capital is estimated on the basis of up to five questions, and here one can get up to 11 points. First, they ask respondents how many foreign languages they know well—depending on the criteria, one can get here from 0 to 2 points. Then they inquire how many own books they have at home on the bookcase - where we have to draw some critical value, for example, at least 30 books (it should be dictated by the specificity of the surveyed environment) - for this question can be gotten 0 or 1 point. In the next stage, respondents are asked to assess themselves as, for example, pupils, students, or employees on a scale from "definitely weak" to "definitely outstanding"- the scale of 0-4. Next topic concerns held formal competences. The authors ask whether the respondents have a driver's license, language certificates, professional qualifications. For each of the documents, one could get 1 point up to 3 points. As before, a list of formal credentials should be constructed in close relation to the studied population. At the very end is positioned a question about achievements, which respondents "can boast". This question was open and one could get one point for this. Here we are looking for success in competitions, contests, events, involvement in important social enterprises.

\section{The Pilot, the Procedure, and the Course}

As with other studies, it is advisable to precede the research by a suitable pilot study. The aim is to refine the tools and procedures, early detection of errors, but also preparation of materials for data analysis. It is important that the groups selected for a pilot testing were similar in terms of the studied traits to the studied target population. The results and experience obtained in this way will facilitate the introduction of necessary amendments in the questionnaire and providing standardized guidelines for the conduct of the test procedure in the target location.

It is reasonable, before the very reaserch take care to have the support and formal approval of the persons responsible for the populations in which the authors study. These can be school principals, rectors and deans of universities, or company managers. 
If possible, we recommend you to conduct the test personally, that is, not through a third party. This allows you immediately to take care of the progress of the research in a consistent manner for all groups and in accordance with the intention of researches and possibly react in case of arising questions or problems.

As in other such cases, the test should be held collectively - that is, the authors study large groups rather than individuals. The test must be preceded by a short introduction that presents persons conducting the test, the purpose of the study, and should contain instructions for completing the questionnaire. Respondents are informed of the possibility of refusing to participate, and are asked to give honest answers and are assured of anonymity.

The whole procedure: Greeting, introduction, completing questionnaires, collecting work, thanks, valediction, should not take more than 30 minutes. Longer working time of respondents is not good for their concentration, and thus reduces the value and quality of answers. It is good practice when interviewers appear in pairs, which not only improves the work, but also prevents unnecessary congestion and confusion. Obviously, you should strive to ensure that the test time is as short as possible.

From the obtained responses, build a database. There are a number of specialized software solutions for this purpose. To a simpler, smaller databases sufficient is MS Excel, to more elaborate purposes Statistica or SPSS. Part of the answer requires coding and interpretation, therefore, for their understanding, should be adopted consistent definitions. During the encoding, also is made pre-selection of answers, resulting in the rejection of questionnaires unsuitable for analysis. Reasons for rejection may be significant gaps in the information, or illegibility.

Properly collected and coded data allow to make analyzes. An effect of the section on the capitals, may be the construction of the scale, from 0 to 31 points $(0-10$ - economic, $0-10$ - social, $0-11$ - cultural). Thus collected data give the authors a comparison of the results of individual respondents and correlating the value of capitals with other studied characteristics.

\section{Conclusions and Analysis Capabilities}

As mentioned earlier, the proposed solution has been already for some years used in social research. As a team, the authors already implemented twice the tool described above and examination procedure.

For the first time during the study conducted in 2013 on the educational plans of young people from one of the Polish regions, the research was conducted at the request of the Rector of the Nicolaus Copernicus University in Torun, and its purpose was, among other things, the diagnosis of the appropriateness of opening a branch of the University in one of the cities in the Central Poland - Grudziądz. The initiator and executor of the project were a six people Monitoring Team for Change in Culture and Education operating at the Faculty of Educational Sciences at the University of Nicolaus Copernicus. The aim of the study was to characterize students of the matriculation classes and youth educational plans of the studied region, particularly focusing on their higher education plans. Empirical data helped to determine the educational plans of subjects and to identify the factors determining them. Estimated was potential for higher education in the study area. Defined were preferences of young people as to the choice of studies and future university, as well as the justifications for declarations in relation to willingness of respondents to take second-cycle studies.

Diagnosed were young people of matriculation and pre-matriculation classes of all secondary schools in Grudziadz, Świecie, Nowe and Łasin. In total, there were 18 schools (secondary and technical schools). Tested were in these schools' 2016 students. 
Collected data during the "surveys in Grudziądz" allowed us to reach clear conclusions, and also helped build the educational-social characteristics of cities and students. Revealed were also very interesting correlations concerning, for example, place of residence and cultural capital. As it turned out, the biggest cities are not the best for the accumulation of this capital, as better are the small towns (20-50 thousand inhabitants). This thesis held up earlier in the literature (Nalaskowski, 1998), now went through empirical confirmation.

An interesting finding is also the one on the gender. Most of the capital "rich" are men. The study revealed a fairly significant difference within the sexes when it comes to the chances of finding itself among the capital "rich". Men, although less numerous in the study population, are in this group more than half (55.6\%). Important here is the fact that men with high symbolic capital are over-represented not only in the techniques, but also in secondary schools, where significantly more women are studying.

The obtained information is allowed to determine the educational plans of surveyed young people. They show that the vast majority, as many as $85.4 \%$ of "the rich", including $47.1 \%$ of women intend to continue their education at the university level. At the same time of "the poor" is going to study every third person (65.2\%), of which nearly two thirds are women.

Socio-economic difficulties of a region are clearly affecting its inhabitants. Research in Grudziądz showed that the situation for young women is more difficult. Focusing on capitals groups of "the poor" and "the rich" confirmed this state of affairs. Young men often represent a higher level of capital wealth, and their educational plans are characterized by the election of prestigious courses of study, as well as faculties of study that give a chance to a secure future in the form of well-paid work in technical professions (Nalaskowski \& Zientarski, 2013).

Another exemplary and interesting proposal was to demonstrate a close and strong correlation between educational plans, and the value of capital. As expected - the higher the symbolic capital, the higher the educational aspirations. But particularly interesting was the fact that the various components of symbolic capital, often correlated with groups of faculties. And so those with particularly high economic capital, often chose business studies and law, and those with particularly high cultural capital choose Humanities and Social Sciences.

Another example of the use of the described methods has just been conducted by the authors of this text research on the identification of students with the state and society. Research in brief called "patriotic", but it is just shorthand for referring to one of the possible attitudes. This research has been carried out since 2014 in several European countries such as Poland, Russia, Georgia, Turkey, Romania, etc. The respondents are students of the third year (age-group about 1992), studying at universities in different faculties. The survey provides information about the attitude of the respondents toward the state and society, as well as tests their knowledge about the country, nation, society. The aim of the research is: (1) knowing the level and extent of identification with the state and society among the young people; (2) diagnosis of levels of capitals: the social, economic, and cultural of the surveyed young people, examination of the relationship between them and the level of identification, which reveal the subjects; and (3) identifying and knowing of the possible relationship between the character of the area of study, and the manifestation of patriotic attitudes.

Just as before, and here for the tools included was the measurement of the value of capitals according to the concept of P. Bourdieu. It is difficult in this case to talk about the final results and outcomes of the measurement, because the research is still in progress - in the following places in Europe, although a preliminary analysis of the data involved, already allow to show some correlation. Perhaps the most interesting 
result is shown strong and statistically significant correlation. People who have a high level of economic capital are more likely to identify with the institution of the state, and have a patriotic attitude. While those with a particularly high social capital and lower economic, most often identify with people, nation than with the institution of the state. Important relationship is also evident between knowledge about the country and society, and the level of symbolic capital. As you might guess - the higher the amount of capital, the better scores on the test of knowledge.

The results presented here are only examples of the use of the strategy described in this study, focusing on some specific research interests. Importantly, the use of the described strategy is perhaps possible in all areas of social research, in which one can refer to the concept of $P$. Bourdieu.

As mentioned in the introduction, the method proposed here has virtually unlimited possibilities of use in the diagnosis of processes, facts and social phenomena. Beyond a reasonable doubt, the skillfully used measurement of capitals, can become an invaluable tool in the hands of persons responsible for policy at all levels, management and implementation of education programs for young people.

\section{References}

Acar, E. (2011). Effects of social capital on academic success: A narrative synthesis. Educational Research and Reviews, 6(6), 456-461.

Białecki, I. (2003). Opportunities for education and social policy: The prospect of equality and social justice. Problems in Childcare and Upbringing, 7(7), 10-13.

Borowicz, R. (2000). The social inequalities in an access to the education: The case of Suwalki. Olecko: Wszechnica Mazurska.

Bourdieu, P. (1986). The forms of capital. In J. E. Richardson (Ed.), Handbook of theory of research for the sociology of education. NewYork: Greenwood Press.

Bourdieu, P. (2007). Outline of a theory of practice. Kety: Wydawnictwo Antyk.

Bowles, S. (2002). Social capital and community governance. Retrieved from http://www.santafe.edu/media/workingpapers/ 01-01-003.pdf

Dejna, D., \& Nalaskowski, F. (2015). The small town as an area of accumulation the educational potential. The Christian Pedagogy, 2(22), 63-78.

Growiec, K. (2011). The social capital: The genesis and social consequences. Warsaw: Acacdemica.

Morgan, S. L. (2000). Social capital, capital goods, and the production of learning. Journal of Socio-Economics, 29, 591-595.

Nalaskowski, A. (1998). The social conditions of the creativity development of the individual. Warsaw: Wydawnictwa Szkolne $\mathrm{i}$ Pedagogiczne.

Nalaskowski, A. (2002). The horizons of education. Kraków: Impuls.

Nalaskowski, F., \& Zientarski, M. (2013). The "rich" and "poor"-Plans of youth education from Grudziądz and surrounding areas and their symbolic capital. Culture and Education, 3(96), 184-200.

Trutkowski, C., \& Mandes, S. (2005). The social capital in small towns. Warsaw: Scholar. 